\title{
Remission of Type 2 Diabetes: A Challenge for Society and Health Care Professionals
}

\author{
Brunella Capaldo ${ }^{1 *}$ and Roberta Lupoli ${ }^{2}$ \\ ${ }^{1}$ Department of Clinical and Experimental Medicine, Federico II University, Italy \\ ${ }^{2}$ Department of Neurosciences, Reproductive and Odontostomatological Sciences, Federico II University, Italy
}

Submission: February 21, 2018; Published: April 03, 2018

*Corresponding author: Brunella Capaldo, Department of Clinical Medicine and Surgery, “Federico II" University, Via S. Pansini 5, 80131, Naples, Italy, Tel/Fax: +39.081.7462302; Email: bcapaldo@unina.it

\begin{abstract}
Type 2 diabetes (T2DM) has become an important public health priority because of its rapidly increasing prevalence driven by an increasing prevalence of overweight/obesity. Given the primary role of obesity in the aetiology of T2DM, weight loss is considered by far the most essential means of controlling the disease. Indeed, growing evidence demonstrates that weight loss achieved either by bariatric surgery (BS) or verylow-calorie diet (VLCD) can lead to reversal of T2DM, at least in patients with short duration of disease. Indeed, a negative energy balance reduces fat content in the liver, in the pancreas as well as in skeletal muscle thus improving insulin action and insulin secretion; this translates into normalization of glucose control. These findings raised great interest regarding the feasibility and the implementation of these therapeutic options in clinical practice. The evidence that T2DM is a potentially reversible condition, at least in an early stage, poses unique challenges to the society as well as to health care providers, who are called to implement these approaches to tackle the epidemic of T2DM.
\end{abstract}

Keywords: Type 2 diabetes remission; Bariatric surgery; Very-low-calorie diet

\section{Introduction}

Type 2 diabetes (T2DM) has become an important public health priority because of its rapidly increasing prevalence, driven by the so called obesogenic enviroment. The latest estimates by the International Diabetes Federation (IDF) indicate that currently there are 415 million patients with diabetes worldwide; this number will escalate to 642 million by 2040 [1]. Given its micro- and macrovascular complications, T2DM represents a heavy burden not only for individuals, but also for health care providers and the society at large.

We have learnt from randomized controlled study that T2DM can be prevented in high risk patients with prediabetes or metabolic syndrome through lifestyle modifications. Reduction in calorie intake (by $500-1000 \mathrm{kcal} /$ day) associated with behavioural interventions and increased physical activity reduce the progression of T2DM by 58\%. More importantly, 10 years after the end of the intervention, there is still a significant reduction in the incidence of T2DM despite some of the weight lost had been regained [2]. Given the primary role of obesity in the aetiology of T2DM, weight loss is considered by far the most essential means of controlling the disease. Indeed, there is growing evidence that T2DM can improve or even be put into remission through weight loss achieved either by bariatric surgery (BS) or very-low-calorie diet (VLCD), thus raising critical issues regarding the feasibility and the implementation of these therapeutic options in clinical practice.

\section{Discussion}

\section{Bariatric surgery and remission of T2DM}

Bariatric surgery yields durable weight loss by altering energy balance through reduction of food intake and/or nutrient absorption. The impact of BS on weight loss is very impressive, with excessive weight loss ranging from $47.5 \%$ after restrictive procedures to $70.1 \%$ after malabsorptive ones [3]. As evidenced in a growing number of publications, BS provides multiple clinical benefits, including improvement/remission of many obesity-related comorbidities, especially T2DM, reduction in cardiovascular disease events and mortality, and improvement of quality of life [4,5]. T2DM remission rate is quite variable among studies depending on patients' characteristics, length of post-surgery follow-up, the criteria used to define remission, and the type of surgical procedure. The agreed criteria to 
define complete T2DM remission are fasting plasma glucose $<100 \mathrm{mg} / \mathrm{dl}$ and/or $\mathrm{HbA} 1 \mathrm{c}<6 \%$ for at least 1 year after surgery in the absence of glucose-lowering pharmacologic treatment; partial T2DM remission is defined as fasting plasma glucose between 100 and $126 \mathrm{mg} / \mathrm{dl}, \mathrm{HbA} 1 \mathrm{C}$ between 6 and $6.5 \%$ and no antidiabetic medication use for at least 1 year; prolonged T2DM remission is defined as a complete remission extending beyond 5 years [6]. As expected, the improvement/remission of T2DM varies according to the surgical technique: $94 \%$ with Biliopancreatic diversion (BPD), 81.6\% with Roux-en-Y Gastric Bypass (RYGB) and 55\% with Adjustable Gastric Banding (AGB) [7]. Interestingly, the improvement of glycemic and metabolic control generally occurs within few days after surgery, long before a substantial weight loss occurs, suggesting that there are weight-independent factors, unique to $\mathrm{BS}$, that promote T2DM remission $[8,9]$.

Numerous observational and randomized controlled trials with follow-up duration up to 5 years have consistently demonstrated the superiority of BS over medical therapy at achieving T2DM remission and improvement of the overall cardiovascular risk profile $[3,10]$. It should be noted, however, that the rate of T2DM remission tend to decrease over time. The median T2DM-free interval after surgery is approximately 8 years (RYGB), while T2DM relapse (restarting antidiabetic medications or fasting plasma glucose $>126 \mathrm{mg} / \mathrm{dl}$ and/or HbA1c $>6.5 \%$ ) may occur in as many as $20-30 \%$ of remitter patients over a period of 6 years [11]. In a large number of T2DM patients undergoing RYGB, up to $70 \%$ experienced complete remission in the first 5 years, but in $35 \%$ of them T2DM relapsed within the subsequent 5 years [11]. Patients at higher risk of T2DM relapse are those with longer duration of disease, older age, more severe $ß$-cell dysfunction, and insulin therapy $[12,13]$.

BS has some risks and complications that need to be assessed together with the aforementioned benefits. Beyond early surgical complications, particularly important are nutritional deficiencies involving vitamins and minerals, which can be prevented through a careful nutritional monitoring and adequate supplementation [14]. Furthermore, the risk of suicide is worryingly higher in bariatric patients as revealed in the longterm follow-up [15].

In the light of clinical evidence, the recent guidelines recognize that $\mathrm{BS}$ is an appropriate treatment for obese people with T2DM and have included this option in treatment algorithms of the disease. They also clearly state that BS should no longer be viewed as a last resort but should be considered earlier in the treatment of T2DM to increase the likelihood of a complete and durable remission of the disease.

\section{Very- low-calorie diet and remission of T2DM}

The beneficial effects of low energy diet on body weight and glucose control have long been recognized. Pathophysiological studies have also clarified the mechanisms through which hyperglycemia can be normalized. As shown by Lim et al. [16], within few weeks of VLCD a significant fall in liver and pancreas fat content occurs with restoration of both hepatic insulin sensitivity and insulin secretion. Recently, several clinical studies have highlighted the potential of VLCD to achieve the remission of T2DM both in adults and in youth [17-19]. The feasibility of achieving reversal of T2DM has been evaluated in a recently published cluster randomized trial (DiRECT) comparing the effects of a structured weight management program versus best practice care on weight loss and T2DM remission in patients with a short duration of disease [20]. The intervention comprised withdrawal of antidiabetic and antihypertensive drugs, total diet replacement 825-853 kcal/day formula diet for 3-5 months), stepped food reintroduction (2-8 weeks), and structured support for long-term weight loss maintenance. At 1-year, 24\% patients in the intervention group lost $15 \mathrm{~kg}$ or more compared to no patients in the control group. T2DM remission was achieved in $46 \%$ patients in the intervention group and $4 \%$ in the control group. Although mean blood pressure at 12 months resulted similar between groups, antihypertensive drugs had been withdrawn in $48 \%$ of participants in the intervention group and in no participants in the control group. Moreover, quality of life improved significantly in the intervention group at 12 months, but was unchanged in the control group. Notably, the study was performed in a primary care setting and the compliance of participants to the intervention program was satisfactory indicating the practicality of this approach in primary care. On the other hand, it must be emphasized that this structured longterm program required are markable investment of human and financial resources.

It is important to consider that the results obtained in the DiRECT study apply to patients with short diabetes duration (up to 6 years) and, therefore, cannot be extended to patients with longer duration of disease and, presumably, more severe $\beta$-cell dysfunction. Furthermore, a longer follow-up is needed to establish the efficacy of this weight management program in keeping DM2 remission over time.

\section{Conclusion}

The message from these studies is that weight loss, achieved either by VLCD or BS, is highly effective in reversing the pathophysiological defects at the root of T2DM. Indeed, a negative energy balance reduces fat content in the liver, in the pancreas as well as in skeletal muscle thus improving insulin action and insulin secretion; this translates into normalization of glucose control. These findings question the general belief that T2DM is a progressive disease requiring increasing number of hypoglycemic agents and, eventually, insulin to face the worsening glucose control. A new vision has emerged indicating that T2DM is a potentially reversible condition at least in an early stage. This piece of evidence calls into action individuals, society and the health care system to implement these approaches and tackle the epidemic of T2DM. 


\section{Current Research in Diabetes \& Obesity Journal}

\section{References}

1. Aschner P (2017) New IDF clinical practice recommendations for managing type 2 diabetes in primary care. Diabetes Res Clin Pract 132 169-170.

2. Klein S, Sheard NF, Pi Sunyer X, Daly A, Wylie Rosett J, et al. (2004) Weight management through lifestyle modification for the prevention and management of type 2 diabetes: rationale and strategies: a statement of the American Diabetes Association, the North American Association for the Study of Obesity, and the American Society for Clinical Nutrition. Am J Clin Nutr 80(2): 257-263.

3. Schauer PR, Mingrone G, Ikramuddin S, Wolfe B (2016) Clinical outcomes of metabolic surgery: efficacy of glycemic control, weight loss, and remission of diabetes. Diabetes Care 39(6): 902-911.

4. Wolfe BM, Kvach E, Eckel RH (2016) Treatment of obesity: weight loss and bariatric surgery. Circ Res 118(11): 1844-1855.

5. Lupoli R, Di Minno MN, Guidone C, Cefalo C, Capaldo B, et al. (2016) Effects of bariatric surgery on markers of subclinical atherosclerosis and endothelial function: a meta-analysis of literature studies.Int J Obes (Lond) 40(3): 395-402.

6. Buse JB, Caprio S, Cefalu WT, Ceriello A, Del Prato S, et al. (2009) How do we define cure of diabetes? Diabetes Care 32(11): 2133-2135.

7. Buchwald H, Estok R, Fahrbach K, Banel D, Jensen MD, et al. (2009) Weight and type 2 diabetes after bariatric surgery: systematic review and meta-analysis. Am J Med 122: 248-256.e5.

8. Griffo E, Nosso G, Lupoli R, Cotugno M, Saldalamacchia G, et al. (2014) Early improvement of postprandial lipemia after bariatric surgery in obese type 2 diabetic patients. Obes Surg 24(5): 765-770.

9. Batterham RL, Cummings DE (2016) Mechanisms of Diabetes Improvement Following Bariatric/Metabolic Surgery. Diabetes Care 39(6): 893-901.

10. Cotugno M, Nosso G, Saldalamacchia G, Vitagliano G, Griffo E, et al. (2015) Clinical efficacy of bariatric surgery versus liraglutide in patients with type 2 diabetes and severe obesity: a 12-month retrospective evaluation. Acta Diabetol 52(2): 331-336.
11. Arterburn DE, Bogart A, Sherwood NE, Sidney S, Coleman KJ, et al. (2013) A multisite study of long-term remission and relapse of type 2 diabetes mellitus following gastric bypass. Obes Surg 23(1): 93-102.

12. Vetter ML, Cardillo S, Rickels MR, Iqbal N (2009) Narrative review: effect of bariatric surgery on type 2 diabetes mellitus. Ann Intern Med 150(2): 94-103.

13. Chikunguwo SM, Wolfe LG, Dodson P, Meador JG, Baugh N, et al. (2010) Analysis of factors associated with durable remission of diabetes after Roux-en-Y gastric bypass. Surg Obes Relat Dis 6(3): 254-259.

14. Lupoli R, Lembo E, Saldalamacchia G, Avola CK, Angrisani L, et al (2017) Bariatric surgery and long-term nutritional issues. World J Diabetes 8(11): 464-474.

15. Dixon JB (2016) Self-harm and suicide after bariatric surgery: time for action. Lancet Diabetes Endocrinol 4(3): 199-200.

16. Lim EL, Hollingsworth KG, Aribisala BS, Chen MJ, Mathers JC, et al. (2011) Reversal of type 2 diabetes: Normalisation of beta cell function in association with decreased pancreas and liver triacylglycerol. Diabetologia 54(10): 2506-2514.

17. Malandrucco I, Pasqualetti P, Giordani I, Manfellotto D, De Marco F, et al. (2012) Very-low-calorie diet: a quick therapeutictool to improve $\beta$ cell function in morbidly obese patients with type 2 diabetes. Am J Clin Nutr 95(3): 609-613.

18. Steven S, Hollingsworth KG, Al Mrabeh A, Avery L, Aribisala B, et al. (2016) Very low-calorie diet and 6 months of weight stability in type 2 diabetes: pathophysiological changes in responders and nonresponders. Diabetes Care 39(5): 808-815.

19. Gow ML, Baur LA, Johnson NA, Cowell CT, Garnett SP (2016) Reversal of type 2 diabetes in youth who adhere to a very-low-energy diet: a pilot study. Diabetologia 60(3): 406-415.

20. Lean ME, Leslie WS, Barnes AC, Brosnahan N, Thom G, et al. (2017) Primary care-led weight management for remission of type 2 diabetes (DiRECT): an open-label, cluster-randomised trial. Lancet 391(10120): 541-551.

Your next submission with Juniper Publishers will reach you the below assets

- Quality Editorial service

- Swift Peer Review

- Reprints availability

- E-prints Service

- Manuscript Podcast for convenient understanding

- Global attainment for your research

- Manuscript accessibility in different formats

( Pdf, E-pub, Full Text, Audio)

- Unceasing customer service

Track the below URL for one-step submission

https://juniperpublishers.com/online-submission.php 